\title{
The Department Chair could have done better...
}

T his scenario is a good example of how important it is to establish relationships up front with incoming recruits and work with our colleagues within and across institutions to establish best practices to support research and optimize animal welfare.

A top priority in this case is ensuring that the veterinarians and staff at Dr. Patel's new university have appropriate training to support the use of desflurane in Patel's cardiac research program. The first thing that the Great Eastern veterinarian should do, as requested by the principal investigator (PI), is to contact the veterinarian at the previous institution to find out what their experience has been with desflurane. The Great Eastern veterinarian should get information regarding how the previous institution managed the desflurane anesthetic support-the PI is bringing all of the equipment, so it is really a matter of training the staff at Great Eastern on how to monitor the anesthesia and be prepared for the different techniques for maintaining appropriate anesthetic depth and managing recovery. Given the unfamiliarity with desflurane the anesthetic support may take more time compared to isoflurane, so cost should be discussed to ensure that the facility recovers the expense of supporting the alternative anesthetic. This can be balanced against the efficiencies of the low-flow rate and decreased environmental pollution when using desflurane ${ }^{1}$.

In addition, Patel needs to provide a more detailed justification as to why the use of desflurane is preferable over isoflurane and how isoflurane would influence the data interpretation. The fact

\section{A WORD FROM USDA}

Under the Animal Welfare Act (AWA) regulations, the research facility is responsible for ensuring all personnel involved in animal care, treatment, and use are qualified to perform their duties. This responsibility can be fulfilled through the provision of training and instruction ${ }^{1}$. The regulations also require an institution to have a program of adequate veterinary care which provides guidance to principal investigators on anesthesia, and ensures the animals receive adequate pre- and post-procedural care in accordance with current established veterinary practices ${ }^{2}$.

In this scenario, a principal investigator accepted a position on the belief he could continue his research using desflurane as the anesthetic. All of the past work was conducted using this anesthetic and to make a change would affect reproducibility. The facility however does not possess the equipment or trained personnel on the use of desflurane. Under the AWA regulations, the attending veterinarian would be responsible for ensuring adequate pain management during the study, and the institution would be responsible for ensuring personnel using desflurane are properly trained ${ }^{1,2}$. As a result, it is in the best interest of the institution, investigator, and veterinarian to work together to identify a solution that balances pain management with research support. The parties may wish to explore conducting the work under contract or in collaboration with an institution that uses desflurane. The Animal Welfare Inspection Guide provides guidance on collaborations, and recommends having an agreement the outlines the responsibilities for animal care, annual reporting, and IACUC oversight ${ }^{3}$.

Betty Goldentyer

Deputy Administrator, Animal Care, APHIS,

USDA, Washington, DC, USA.

e-mail:betty.j.goldentyer@usda.gov

Published online: 25 February 2020

https://doi.org/10.1038/s41684-020-0485-5

References

1. Title 9 Chapter 1 Subchapter A $\$ 2.32$ (a)

2. Title 9 Chapter 1 Subchapter $A \$ 2.33$ (b) (4-5)

3. Animal Welfare Inspection Guide Chapter 7 Section 7.3 Contracted Research or Projects that Involve Multiple Registrants https://www.aphis.usda.gov/animal_welfare/ downloads/Animal-Care-Inspection-Guide.pdf that all historical data are with desflurane is not adequate justification to continue the use of desflurane if in fact isoflurane is a comparable or even superior anesthetic. All PIs need to be open to considering refinements suggested by the veterinarian and the IACUC, and there should be strong scientific justification for the continued use of desflurane.

If we assume that the use of desflurane is scientifically justified to be necessary and appropriate, then the IACUC and the veterinarian should work to incorporate this new anesthetic into the local regimen. They should identify individuals on staff to be trained to support the desflurane anesthesia. A pilot study would not be necessary if there is adequate scientific justification. All relevant members of the animal care and use program should work together to get the PI's studies implemented as soon as possible.

Establishing good collaborative relationships with investigators is the critical foundation for successful management of animal care at any institution.

Any costs associated with training Great Eastern staff should be paid by the department Chair who assured Patel it would be no problem to use an anesthetic that was new to the institution. At some point, there must be an in-depth conversation with the Chairperson about how this would have been handled more appropriately if the correct individuals were involved up front. Any recruitment of PIs whose research uses live animals should include a meeting and conversation with both a veterinarian and a representative of the IACUC (the IACUC Chair or Director of IACUC office, depending on the structure of the institution) during the interview process to facilitate integration into a new research community.

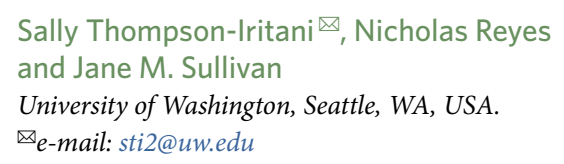

